\title{
Challenges of Adopting Human-Centered Intelligent Systems: An Organizational Learning Approach
}

\author{
Fons Wijnhoven
}

\begin{abstract}
Clinical decision support systems (CDSSs) are human-centered intelligent systems (HCISs) that use codified medical expertise or large data sets for medical decision recommendations. Most analytical CDSS that exploit the opportunities of large data sets and analytic technique remain within a research and development environment and lack adoptions in clinical contexts. To understand this, we analyse CDSS adoption as an organizational learning process. We apply a model of organizational learning on the case of an analytical CDSS implementation which analyses medical data to predict the probability on sepsis for prematurely born babies to support the physicians' decision-making on ministering antibiotics. In our discussion, we next compare our case findings with possible organizational learning challenges for the adoption of other (medical) HCISs and we draw consequences for projects of HCIS adoption in organizations.
\end{abstract}

\section{Introduction}

Clinical decision support systems (CDSSs) are systems for clinical decision making [1]. A CDSS can contain multiple techniques to support medical decision-making, like visualization of clinical data [2, 3], zooming, sorting and filtering to deep dive in specific sections of relevant patient data, images and video recordings [4-6] and analysis of data from multiple sources like the patient medical record and genomic data for diagnosis and treatment selection [7]. Natural language processing (NLP) can also be used to extract the meaning from natural language text notes in medical records [8-10]. More complex forms of technology involve machine learning, i.e. methods that can automatically detect patterns in data [11]. CDSSs like Watson for Oncology incorporate a form of prescriptive analytics by ranking treatment alternatives along predicted effectiveness for a given diagnosis with the support of mining knowledge from 600 medical journals, hundreds of different medical data sources

\footnotetext{
F. Wijnhoven $(\bowtie)$

University of Twente, Enschede, The Netherlands

e-mail: a.b.j.m.wijnhoven@utwente.nl

(C) The Editor(s) (if applicable) and The Author(s), under exclusive license to Springer Nature Singapore Pte Ltd. 2021

A. Zimmermann et al. (eds.), Human Centred Intelligent Systems, Smart Innovation, Systems and Technologies 189, https://doi.org/10.1007/978-981-15-5784-2_2
} 
Table 1 Analytics techniques in clinical DSS [13]

\begin{tabular}{l|l}
\hline Technique & Healthcare application examples \\
\hline Cluster analysis & Detecting high-risk obesity groups \\
\hline Machine learning & $\begin{array}{l}\text { Predicting disease risk; Detecting } \\
\text { epidemics }\end{array}$ \\
\hline Neural networks & $\begin{array}{l}\text { Diagnosing chronic diseases; Prediction } \\
\text { patients' future diseases }\end{array}$ \\
\hline Pattern recognition & Improving public health surveillance \\
\hline
\end{tabular}

and statistical evidence [12]. Mehta and Pandit summarize analytic techniques for CDSS in Table 1 [13].

Mehta and Pandit [13] state that all the studies they found describe, develop and test a model or algorithm to show its added value, however, do not mention anything about implementation. Mehta and Pandit [13] suggest that a reason for the lack of these CDSS implementations is that the current body of literature does not provide adequate quantitative evidence that these techniques can be trusted by medical practitioners in their clinical use. Also other review articles state that different from research contexts, medical clinical contexts have very high levels of ethical, legal and reasoning transparency demands that are difficult to meet in practice [14]. A lack of trust in the systems recommendations is also an important reason for not adopting CDSS by physicians [14]. Highly problematic is also medical journalists claim that Watson for Oncology's training resulted in a bias towards Memorial Sloan Kettering physicians' preferences, which is not unlikely because these physicians were involved in the development of Watson for Oncology $[15,16]$. Other technology companies such as Google and Microsoft have developed similar CDSS [17]. These systems remain within the R\&D environment and have not been implemented in clinical practice either [18].

Despite these adoption problems, McNut et al. [19] and Rumsfeld et al. [20] state that analytics has great promises for the fields of oncology and cardiovascular diagnoses and treatments, respectively, but that these promises only can become realized when sufficiently large and reliable datasets are available. Unfortunately, this is difficult to achieve. For realizing such large data sets hospitals will have to share their data [21-23]. Lack of systems interoperability and different data taxonomies prevent such inter-hospital data sharing and thus further structuring and standardization of systems and data is needed [7, 24]. NLP of informal language that describes patient's status and medical doctor's thoughts are a new opportunity for CDSS. These data are available via patient medical records, but accessibility of these data for analytics is legally and ethically complicated and classifying natural language in medical terminology is still not very reliable [25]. Besides of all these analytical workflow challenges, CDSSs are also difficult to use from the perspective of the medical practitioner. Medical practitioners are especially concerned regarding the accuracy of classifications and predictions when the data sets are too small [26-30] and the algorithms used are non-transparent or incomprehensible [31]. Intransparency of algorithms may result in feelings of loss of reasoning control which is unacceptable for medical 
professionals during their diagnosis and treatment decision-making [14, 32, 33]. Experiences with unreliable registrations in patient files also do not contribute much to trust in CDSS [23]. Many of the reasons for not trusting and resisting CDSS are thus not technical or psychological (i.e. the medical professionals risk perception) but are rooted in ethical, legal and managerial requirements being insufficiently met $[34,35]$. Finally, also the actual realization of a clinical CDSS requires new knowledge, expertise and training in use and interpretation of results and new IT personnel that can give the proper support and use conditions. Given the increasing costs of healthcare these extra resources are difficult to fund.

In this article, we present an organizational learning approach to these HCIS adoption challenges, motivated by the belief that the most fundamental change a HCIS can bring to professionals is that it may change their competences of learning and finding knowledge and solutions for problems that the average person will not be able to handle.

Following a classic article on organizational learning [36], we describe organizational learning as a cyclic process of knowledge development. This process starts with externalizing and codifying tacit knowledge of individuals and placing this on a human independent medium like and information system. Second, this explicit and codified knowledge can be combined with other explicit knowledge, e.g. by the creation of data warehouses and extensive rule-based expert systems. Third, the more advanced combined knowledge can produce advanced recommendations to decision makers, but the decision maker must be able to internalize the recommendation by combining it with his or her personal experiences, values, and skills, i.e. tacit knowledge, to be able to take the responsibility for the decision. Finally, professionals often discuss problems and recommendations with colleagues for verification or group decision-making, or they share the decision, recommendation and cases with colleagues after the decisions to learn from each other or develop new standards and guidelines. See Fig. 1 for the Nonaka model, which presents this learning process as a continuous cycle.

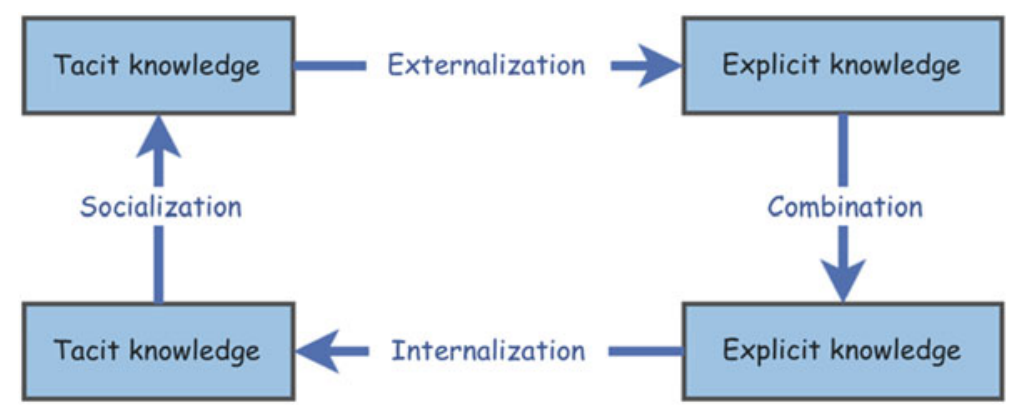

Fig. 1 Nonaka's SECI model. Remake after [36] 
This brings us to our research questions:

1. What challenges can happen in the externalization stage of HCIS adoption?

2. What challenges can happen in the combination stage of HCIS adoption?

3. What challenges can happen in the internalization stage of HCIS adoption?

4. What challenges can happen in the socialization stage of HCIS adoption?

The next section explains the methodology for detecting relevant adoption challenges. Section 3 presents our results and Sect. 4 discusses these results in the broader contexts of other variants of HCIS systems. Lastly, Sect. 5 entails the conclusion and reflects about further research.

\section{Methodology}

As stated in the research questions, this research wants to register the organizational learning challenges for a concrete implementation of a CDSS. To realize this, we use a rich case description in which key stakeholders were interviewed regarding their view on the adoption of an HCIS. We first describe this case in the following subsection, after which we go in details about the stakeholders and our analysis.

\subsection{The BD4SB Case}

The Utrecht University Medical Centre (UMCU) initiated the Applied Data Analytics in Medicine (ADAM) project in spring of 2017 to make healthcare more personalized with analytics in collaboration with external partners such as Siemens, Philips, SAS and Accenture. This is a hospital-wide project with a special team of clinicians and data scientists. ADAM enables pilots from four departments within the UMCU, among which the BD4SB pilot within the Neonatology Department that cares for premature babies. Babies that are born too early are sensitive to infection. Regretfully, treatment with invasive procedures, such as intravenous lines, blood samples and ventilation are all potential entry points for bacteria and add to the risk of illness. The Neonatology Department wants to know as early as possible when a patient will become ill and what treatment is most appropriate. The current healthcare process is as follows: (1) the physician suspects an infection (e.g., skin colour change, blood pressure or temperature instability), (2) the physician takes a blood culture, (3) this blood culture is examined for bacteria in the laboratory and (4), when the culture is positive, the blood is examined by Gram staining (bacteria are coloured to make them visible under the microscope to identify the species). This process can take up to $48 \mathrm{~h}$ which can be crucial in the development of the infection and the administration of antibiotics. Not administering antibiotics must be considered carefully since sepsis has negative consequences for the patient; however, administering antibiotics can 
also have negative consequences such as an increased chance on other diseases as asthma, cancer, intestinal diseases or obesity.

The BD4SB CDSS aims to support the physicians when they consider administering antibiotics. The CDSS focuses on predicting with a minimum of false negatives. False negatives are the most dangerous situations because the advice is not to give antibiotics when it is needed. The BD4SB CDSS uses different data sources from the database of neonatology which consists of 6,000 children born between 24 and 32 weeks. These data originate from several systems whose data must be integrated and prepared within data management before analysis. The model development method applied for analysis is the 'gradient boosting' technique of predictive machine learning.

\subsection{Data Collection and Analysis}

Twenty-three-key expert stakeholders were interviewed in a semi-structured way about challenges for the CDSS implementation. The unscripted narrative enabled the researcher to explore the respondents' expertise. We categorize the given statement in this article as representative for adaption challenges per organizational learning process in Sect. 3. The stakeholders and their functions are given in the case descriptions. The respondents were visited or approached at an event.

\section{Results: BD4SB Adoption Challenges}

This section presents data for the four organizational learning categories of Fig. 1.

\subsection{Externalization Challenges}

Externalization is the transformation of tacit knowledge to explicit knowledge that can be stored on a person-independent medium. The term 'knowledge' is used here in a very broad sense in the many ways that people say to know something, and thus includes facts and figures, theories and explanations, methods and skills and experiences [37]. We found many statements from which we can say that the CDSS is adoption is challenging.

The data quality in healthcare in general suffers from inaccurate registrations. The inaccurate registration is partially caused by the high registration load within healthcare and users' inability to see the benefits of registration. R5 (professor health informatics) says: "I believe that a lot of professionals are not satisfied with the registration load...." R1 (ex-chairman board of AMC) says: "There is a tension 
between how we can standardize the systems input and how can we keep the input requirements user friendly."

Even when the data would be available and correct, a lot of data processing and analytics is needed which unfortunately is not always compliant with $\mathrm{CE}^{1}$ quality norms. R11 (ex-physician and data scientist UMCU) says: "Firstly, some data producing machines within the UMCU are validated and CE approved for research and not for healthcare which is needed for BD4SB, a new CE approval of the data warehouse is required to realize this transition. Secondly, data source HIX (EHR) is currently updated once a day, however, only converted to usable input for analytics once a week."

Physicians also hold professional expectations that make them hesitant to work with a CDSS. R20 (ex-physician and analytical entrepreneur) says: "CDSS technology enforces strict working according to guidelines and thus may deprive physicians from their sense of added value. This perhaps is the single biggest reason why working with CDSS technology is so slowly being adopted - it makes physicians feel less valuated."

This hesitation is not necessarily a defensive or conservative attitude, but could be based on sincere professional doubts regarding a positive cost-benefit relation between knowledge externalization and contributions to medical care. R17 (Business engineer CDSS developer) says: "It is not clear if the improvement of care is worth the costs of data scientists, medical trial, infrastructure and maintenance of the BD4SB project."

One of these doubts may also be based on the infeasibility of some essential parts of a CDSS. The most optimal design for a clinical evaluation study is a randomized control trial (RCT), but R4 (clinical owner BD4SB) says: "The best way would be a randomized experiment where half will be exposed to the algorithm and the other half not. This is seriously hard, randomizing thousands of patients and the algorithm might be only suitable for our own population, we have to look at how we can show the clinical relevance without a randomized study."

\subsection{Combination Challenges}

Combination is the process of bringing different externalized parts (like databases and applications) together in one larger system.

$\mathrm{R} 12$ (clinical owner BD4SB) says: "All the data the CDSS requires can be extracted from the hospital wide critical data layer for analytical proceedings by means of an API call. This data layer collects patient specific data from each individual by means of patient ID. We need this step or else we will still be looking at retrospective data. This data layer is currently under construction, technologically feasible, however, realization depends on commitment and budget."

\footnotetext{
${ }^{1} \mathrm{CE}$ stands for Conformite Européenne and is the European Union quality certification label.
} 
This layer would automatically collect, integrate and prepare the data from different data sources, but there is a lack of data sharing protocols. R8 (Senior technical consultant SAS) says: "Data protocols describe among other things what data was used, where the algorithm was developed, which version it was and how it should be utilized. This increases the controllability and auditability. They are not used currently. Every system generates its own data in a way that is most easy for this system. Which is not wrong of course. Only when you want to join the data from these systems, you might realize that you should have done it in a different manner."

.....and data are poorly shared among different applications as R12 (clinical owner BD4SB CDSS) says: "There is no automated process that collects data for analysis and makes it available to other solutions, all the data is still in its original source and has to be extracted and integrated manually by somebody to enable the following analysis."

\subsection{Internalization Challenge}

Internalization is the process of humanly interpreting knowledge, insights and recommendations given by a system. For making recommendations acceptable for decision makers, they have to rely on transparent data analysis procedures.

R10 (ex-physician and sales manager SAS) says: "The analytical process often requires transparency. For example: which algorithm was used six months ago, which data was used and who entered or changed the data, were they entitled to do so, does the data sources supply the required quality or how it was analysed. If a physician stands in front of a judge, he/she must be able to exactly explain how the process was executed."

However, the system must be compliant with the standards for CE approval.

$\mathrm{R} 12$ (clinical owner BD4SB): "However, the system still misclassifies some patients because they had other symptoms or something special, only 6 of the 500 children. This is not much, however, if all 6 babies die, this is hard to explain, of course." And R13 (Program manager ADAM) says: "It does not matter if the algorithm says to intervene or not to intervene, the physician is always responsible and therefore is hesitant in using these systems." And R10 (ex-physician and senior sales executive healthcare SAS) says: "When a complaint is made, a hospital/clinician has to justify every step within the treatment process."

\subsection{Socialization Challenges}

Socialization is the process of sharing tacit knowledge among people. This tacit knowledge may be any insight or experience that is not fully formally defined and not made person-independent and may have a role before externalization but also have a role in the process of human decision-making. 
R7 (healthcare director SAS): "Hospitals have another problem. A cultural problem, the physicians are too distant from the IT department." Involving physicians within the development stage of the analytical CDSS will be beneficial for trust and acceptancy of an analytical CDSS. Therefore, R11 (ex-physician and data scientist UMCU) says: "It is important to bring along a group of physicians within this process or else you will get the "not invented by me syndrome'."

Whereas the full responsibility for medical decisions lies with the physician, there is a certain kind of moral responsibility that lies with the developer. R16 (Ethicist and member medical ethical commission, UMCU) says: "If an algorithm makes a mistake or if a device makes a mistake, the developer is partially responsible since they built this into the algorithm, when there is a causal link between the mistake and the algorithm."

The Medical Ethical Assessment Committee (METC) has no clear legal framework for a predictive algorithm within the WMO. R11 (physician and data scientist UMCU) says: "CE approval requires the description of risks when it goes wrong for which you need a test period. To execute this test period, you need to deliver a risk assessment to the METC, this is a vicious circle."

Legislation only states to execute a good risk assessment but there is no specification on requirements for predictive algorithms within such an assessment (R17, business engineer developer CDSS). According to R19 (inspector e-health), the EU is currently developing norms for artificial intelligence which are also applicable to analytical CDSS. These norms could also give more foundation to the METC within the medical trial approval since it provides a greater understanding within a legal framework. The interpretation of the MDR is still under construction according to R22 (global clinical director notified body): "Dekra has updated its procedures to the new legislation on European level, the MDR. The inspection healthcare (IGJ) and representatives of other EU member states validated the new MDR procedures during joined assessments. Very often during these joined assessments the EU member state representatives had different interpretations of the regulations. Hence, it took 7 years to write the new legislation, then you have a meeting with representatives of a notified body from several countries and the healthcare inspection (IGJ) and they are still discussing what the MDR exactly says. The new legislation leaves room for interpretation probably because a $100 \%$ alignment of all EU member states is difficult to reach."

Including a regulatory expert in the $R \& D$ team might be beneficial to the $C E$ approval process preparation of the BD4SB project team. R22 (Clinical CEO notified body, Dekra) says: "A regulatory professional within a $R \& D$ team involved from step one can think of what Notified Body or food and drug administration (FDA) market approval conditions are. Large companies see this and include a regulatory professional from the concept stage."

Thus, the socialization process is not just among only physicians and their teams, but also with other technical, ethical and legal stakeholders, who all mutually have to be able to understand each other. 


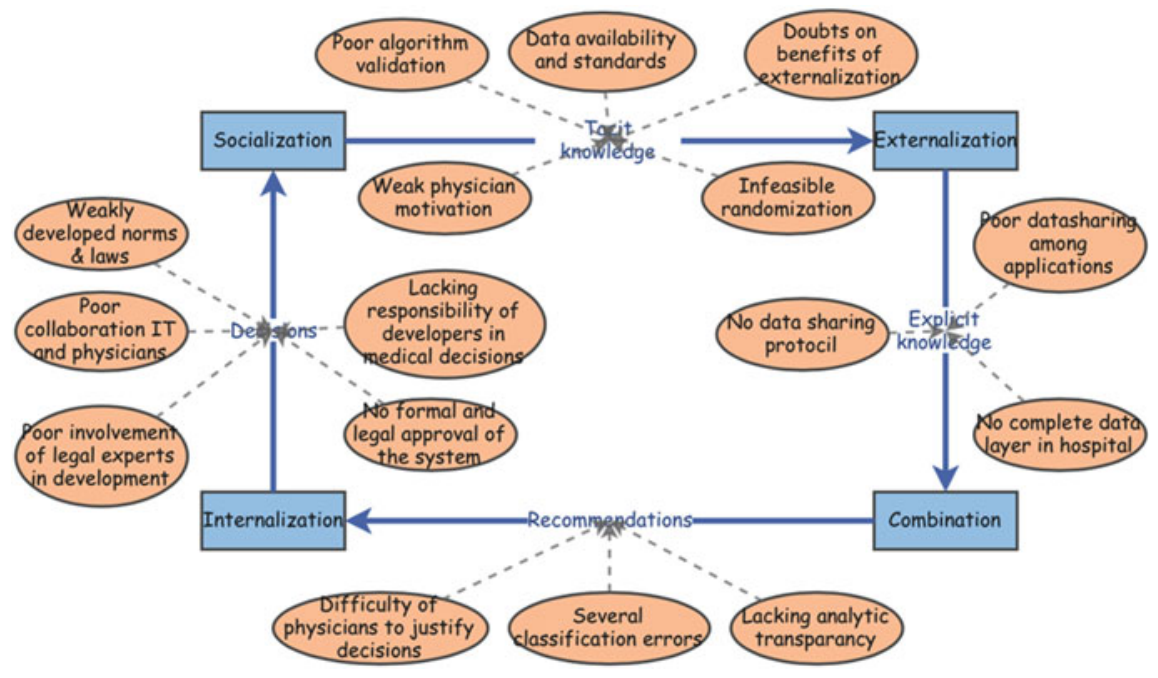

Fig. 2 Summary of CDSS development challenges

\subsection{Case Summary and Discussion}

Figure 2 summarizes the BD4SB development challenges as boxes and ovals, respectively, within the analytical CDSS development project. Nearly all challenges influence the difficulty of physicians to justify their diagnostic or treatment selection decisions and could result in less motivation from their side to engage in CDSS.

\section{Discussion}

In this section, we aim at finding general lessons for the development of HCIS on basis of this case. In grounded theory development this is named the formalization of theory [38] by generalizing over key factors. As key factors we identify the different knowledge types that CDSS/HCIS system can handle and different environments outside the context of hospitals.

In Sect. 1, we introduced Minger's classification of "knowledge": facts and figures, theories and explanations, methods and skills and experiences [37]. The CDSS case discusses knowledge as facts and figures so that physicians know exactly and more quickly the status of a patient. This type of classification knowledge is not the knowledge that physicians find in their books, their methods or skills and experiences. A CDSS that would focus on delivering knowledge as theories and explanations is different and has a different organizational learning process organized in academic research, text books and medical journals. Such knowledge can be stored in expert systems and medical guidelines, although experiences has shown that developing and 
maintaining such a system is complex and time-consuming [39, 40]. An interface with publishers' content for very fast search, not just on key terms but especially on basis of diagnostics data as input could be very useful HCIS application here. "Methods \& skills" knowledge is hard to place in a CDSS, although medical guideline systems do give some indications of what to do when, but these systems are of course not the professional skills themselves that are part of tacit knowledge developed through much training. Medical guideline systems, however, do need a very extensive and active maintenance process. This is possible only if the governance structure for such a system is well developed and well managed.

"Experiences" knowledge can be shared nowadays easily via social media and problem-solving fora. There may not be many active platforms for physicians who exchange experiences mainly via conferences and inter-colleague consultations, which also enable high-level socialization. Very interestingly, patients do share experiences, especially about rare diseases, which enable professionals to mine insights [41].

There seems to be a fundamental difference between systems that create or manage rules and systems that classify. Rule-based systems bundle and combine experiences, methods, theories and explanations in what if expressions. Classification systems only process facts and figures to state in what state someone or something is. Depending on these classifications, the rules prescribe what to do, and so they are related, but analytical CDSS (like the BS4SB case) are only classifiers.

Whereas expert systems or rule-based systems are transparent when rules are created and maintained by people, analytic systems apply algorithms that are often black boxes to their users. The lack of insights in how the algorithms make their conclusions has a much higher level of risk perception than the manual rule defining systems.

Question remains if this would be valid for non-hospital settings. Clearly, the professional context of a physician is different from fields like manufacturing, services, education, construction or logistics. How different? They all may use large data sets, explicit expert knowledge, experience-sharing platforms and methods and skills. So, is it all really that different? Maybe less regulated by law and quality norms, as people's life do not depend that much on these services but highly a matter of business or financial risk that are just part of the regular business game. Therefore, we identify two critically different variables that determine the adoption challenges of HCIS: (1) level of impact on life versus (2) risks of lack of transparency of reasoning.

Regarding impact of life, the dichotomy between low and high is of course a gross simplification and many nuances on this scale are needed. Whereas in the extreme low impact on life people do not care that computers or people are making decisions for them, e.g. the cases of the application of administrative rules, in the other extreme the decisions may be a matter of life, death or happiness, as in many medical decisions, personnel recruitments or work evaluations. On this scale, in the middle are decisions that apply human fairness principles, i.e. the decisions should handle the interests of women, ethnic minorities or other demographic characteristics in a fair (Table 2). 
Table 2 HCIS adoption challenge dimensions and example systems

\begin{tabular}{l|l|l|l}
\hline \multicolumn{2}{l|}{} & \multicolumn{2}{l}{ Impact on life } \\
\cline { 3 - 4 } & $\begin{array}{l}\text { Low (e.g. business } \\
\text { services) }\end{array}$ & High (e.g. medical) \\
\hline $\begin{array}{l}\text { Transparency of } \\
\text { reasoning }\end{array}$ & $\begin{array}{l}\text { Low (e.g. data } \\
\text { mining) }\end{array}$ & $\begin{array}{l}\text { Customer segmentation } \\
\text { algorithms }\end{array}$ & Analytic CDSS \\
\cline { 2 - 4 } & $\begin{array}{l}\text { High (e.g. rules } \\
\text { applied) }\end{array}$ & $\begin{array}{l}\text { Banking rules loan } \\
\text { application system }\end{array}$ & $\begin{array}{l}\text { Medical expert } \\
\text { (guidelines) system }\end{array}$ \\
\hline
\end{tabular}

Regarding the reasoning transparency dimension, low and high is again lacking nuances. The application of rules may need a clear classification of cases, where sometimes the class boundaries are less clearly to be identified. The BD4SB case is an example here, where it is not sure if a baby had sepsis, but when it has, the rule is clear and antibiotics should be administered. Social programmes against poverty meet a similar problem. Whereas in these programmes money is available for people lacking financial means, one may debate if these funds should be available for university students with unemployed middle-class parents. Algorithms that perform the classifications but also the data themselves can be biasing the cut-offs for the application or non-application of rules.

In both cases, HCIS researchers and users should be aware of fairness impacts and algorithmic biases, which means that HCIS with social impact need the influence of affected stakeholders in a democratic way.

\section{Conclusions}

This study aimed at understanding the challenges of HCIS adoptions as an organizational learning process. We found many challenges summarized in Fig. 2 for a clinical DSS, and we brought this case in the contexts of fairness and biases, where the CDSS case is on the high risks of life and low transparency of reasoning, making adoption of CDSS not a complex case. Given the high responsibility of physicians in medical decision making, resistance in the use of CDSS may be possibly wise abstention [42]. The lack of trust is not just rooted in a technical or organizational difficulty, but especially is rooted in legal and interorganizational frameworks that are not well in place yet. A new kind of decision-making process must also be developed with new roles for physicians, patients and CDSS developers. Although business applications of HCISS, like product recommenders and customer services management, may have less a context of life or death, companies and governments also have to treat their customers fair and non-biased. This also implies that HCIS systems development should not be the sole work of knowledge engineers but require the explicit and intensive involvement of business people who can translate the norms of handling customers fair and non-biased. HCIS systems therefore should be validated also on 
fairness and absence of bias. This may require the involvement of representatives of the people affected by these systems.

Acknowledgements The case for this article is based on data collected by Rick Klein Koerkamp. This article, which gives an analysis to the more general domain of HCIS, however, is the full responsibility of the author.

\section{References}

1. Marakas, G.: Decision support systems in the 21st century. ACM SIGSOFT Softw. Eng. Notes 27, 104 (1999)

2. Turkay, C., Jeanquartier, F., Holzinger, A., Hauser, H.: On Computationally-Enhanced Visual Analysis of Heterogeneous Data and Its Application in Biomedical Informatics, pp. 117-140 (2014)

3. Wongsuphasawat, K., Guerra Gómez, J.A., Plaisant, C., Wang, T., Taieb-Maimon, M., Shneiderman, B.: LifeFlow. In: CHI 2011 Proceedings of the SIGCHI Conference on Human Factors in Computing Systems, pp. 1747-1756. ACM, New York (2011)

4. Korotkov, K., Garcia, R.: Computerized analysis of pigmented skin lesions: a review. Artif. Intell. Med. 56, 69-90 (2012)

5. Faggella, D.: Where healthcare's big data actually comes from. Tech Emerg, 11 (2018)

6. Exastax: Top Five Use Cases of Tensorflow. https://www.exastax.com

7. Raghupathi, W., Raghupathi, V.: Big data analytics in healthcare: promise and potential. Heal. Inf. Sci. Syst. 2, 3 (2014)

8. Holzinger, A., Geierhofer, R., Mödritscher, F., Tatzl, R.: Semantic information in medical information systems: utilization of text mining techniques to analyze medical diagnoses. J. Univ. Comput. Sci. 14, 3781-3795 (2008)

9. Ivanović, M., Budimac, Z.: An overview of ontologies and data resources in medical domains. Expert Syst. Appl. 41, 5158-5166 (2014)

10. Sivarajah, U., Kamal, M.M., Irani, Z., Weerakkody, V.: The university of bradford institutional repository. J. Bus. Res. 70, 263-286 (2017)

11. Murphy, K.P.: Machine Learning-A Probabilistic Perspective. Table-of-Contents (2012)

12. Somashekhar, S.P., Sepúlveda, M.J., Puglielli, S., Norden, A.D., Shortliffe, E.H., Rohit Kumar, C., Rauthan, A., Arun Kumar, N., Patil, P., Rhee, K., Ramya, Y.: Watson for Oncology and breast cancer treatment recommendations: agreement with an expert multidisciplinary tumor board. Ann. Oncol. 29, 418-423 (2018)

13. Mehta, N., Pandit, A.: Concurrence of big data analytics and healthcare: a systematic review. Int. J. Med. Inform. 114, 57-65 (2018)

14. Eberhardt, J., Bilchik, A., Stojadinovic, A.: Clinical decision support systems: potential with pitfalls. J. Surg. Oncol. 105, 502-510 (2012)

15. Petitjean, F.: IBM Watson kiest foute kankerbehandeling

16. Ross, C.: IBM's Watson supercomputer recommended 'unsafe and incorrect' cancer treatments, internal documents show. https://www.statnews.com/wp-content/uploads/2018/09/IBMs-Wat son-recommended-unsafe-and-incorrect-cancer-treatments-STAT.pdf

17. Nijhof, K.: Watson for oncology maakt forse stappen

18. Electronics, A., Batra, B.G., Queirolo, A., Santhanam, N.: Artificial intelligence: the time to act is now, 1-16 (2018)

19. McNutt, T.R., Moore, K.L., Quon, H.: Needs and challenges for big data in radiation oncology. Int. J. Radiat. Oncol. Biol. Phys. 95, 909-915 (2016)

20. Rumsfeld, J.S., Joynt, K.E., Maddox, T.M.: Big data analytics to improve cardiovascular care: promise and challenges. Nat. Rev. Cardiol. 13, 350 (2016) 
21. Dinov, I.D.: Methodological challenges and analytic opportunities for modeling and interpreting Big Healthcare Data. Gigascience 5, 12 (2016)

22. Peek, N., Holmes, J.H., Sun, J.: Technical challenges for big data in biomedicine and health: data sources, infrastructure, and analytics. Yearb. Med. Inform. 23, $42-47$ (2014)

23. Salas-Vega, S., Haimann, A., Mossialos, E.: Big data and health care: challenges and opportunities for coordinated policy development in the EU. Heal. Syst. Reform. 1, 285-300 (2015)

24. Asokan, G.V., Asokan, V.: Leveraging "big data" to enhance the effectiveness of "one health" in an era of health informatics. J. Epidemiol. Glob. Health 5, 311-314 (2015)

25. Auffray, C., Balling, R., Barroso, I., Bencze, L., Benson, M., Bergeron, J., Bernal-Delgado, E., Blomberg, N., Bock, C., Conesa, A.: Making sense of big data in health research: towards an EU action plan. Genome Med. 8, 71 (2016)

26. Budhiraja, R., Thomas, R., Kim, M., Redline, S.: The role of big data in the management of sleep-disordered breathing. Sleep Med. Clin. 11, 241-255 (2016)

27. Cox, M., Ellsworth, D.: Application-controlled demand paging for out-of-core visualization. In: Proceedings. Visualization 1997 (Cat. No. 97CB36155), pp. 235-244. IEEE (1997)

28. Geerts, H., Dacks, P.A., Devanarayan, V., Haas, M., Khachaturian, Z.S., Gordon, M.F., Maudsley, S., Romero, K., Stephenson, D., Initiative, B.H.M.: Big data to smart data in Alzheimer's disease: the brain health modeling initiative to foster actionable knowledge. Alzheimer's Dement. 12, 1014-1021 (2016)

29. Kruse, C.S., Goswamy, R., Raval, Y.J., Marawi, S.: Challenges and opportunities of big data in health care: a systematic review. JMIR Med. Inform. 4, e38 (2016)

30. Szlezak, N., Evers, M., Wang, J., Pérez, L.: The role of big data and advanced analytics in drug discovery, development, and commercialization. Clin. Pharmacol. Ther. 95, $492-495$ (2014)

31. Maia, A.-T., Sammut, S.-J., Jacinta-Fernandes, A., Chin, S.-F.: Big data in cancer genomics. Curr. Opin. Syst. Biol. 4, 78-84 (2017)

32. Holden, R.J., Karsh, B.T.: The technology acceptance model: its past and its future in health care. J. Biomed. Inform. 43, 159-172 (2010)

33. Maillet, É., Mathieu, L., Sicotte, C.: Modeling factors explaining the acceptance, actual use and satisfaction of nurses using an Electronic Patient Record in acute care settings: an extension of the UTAUT. Int. J. Med. Inform. 84, 36-47 (2015)

34. Andreu-Perez, J., Poon, C.C.Y., Merrifield, R.D., Wong, S.T.C., Yang, G.Z.: Big data for health. IEEE J. Biomed. Health Inform. (2015)

35. Abouelmehdi, K., Beni-Hssane, A., Khaloufi, H., Saadi, M.: Big data security and privacy in healthcare: a review. Procedia Comput. Sci. (2017)

36. Nonaka, I.: A dynamic theory knowledge of organizational creation. Organ. Sci. 5, 14-37 (1994)

37. Mingers, J.: Management knowledge and knowledge management: realism and forms of truth. Knowl. Manag. Res. Pract. 6, 62-76 (2008)

38. Glaser, B.G., Strauss, A.L.: The Discovery of Grounded Theory: Strategies for Qualitative Research. Adeline, Chicago, Illinois (2009)

39. Bensoussan, A., Mookerjee, R., Mookerjee, V., Yue, W.T.: Maintaining diagnostic knowledgebased systems: a control-theoretic approach. Manag. Sci. (2008)

40. Grosan, C., Abraham, A.: Rule-based expert systems. Intell. Syst. Ref. Libr. (2011)

41. Dirkson, A., Verberne, S., Van Oortmerssen, G., Gelderblom, H., Kraaij, W.: Open knowledge discovery and data mining from patient forums. In: 2018 IEEE 14th International Conference on e-Science (e-Science), pp. 397-398. IEEE (2018)

42. Althuizen, N., Reichel, A., Wierenga, B.: Help that is not recognized: harmful neglect of decision support systems. Decis. Support Syst. 54, 713-728 (2012) 